\title{
A Logic of Directions
}

\author{
Heshan Du ${ }^{1}$, Natasha Alechina ${ }^{2}$ and Anthony G. Cohn ${ }^{3}$ \\ ${ }^{1}$ University of Nottingham Ningbo China \\ ${ }^{2}$ Utrecht University, Netherlands \\ ${ }^{3}$ University of Leeds, UK \\ heshan.du@nottingham.edu.cn,n.a.alechina@uu.nl, a.g.cohn@leeds.ac.uk
}

\begin{abstract}
We propose a logic of directions for points $(L D)$ over 2D Euclidean space, which formalises primary direction relations east $(E)$, west $(W)$, and indeterminate east/west $\left(I_{e w}\right)$, north $(N)$, south $(S)$ and indeterminate north/south $\left(I_{n s}\right)$. We provide a sound and complete axiomatisation of it, and prove that its satisfiability problem is NP-complete.
\end{abstract}

\section{Introduction}

This work is motivated by the problem of matching spatial objects represented in different geospatial datasets and verifying the consistency of matching relations. A matching relation states that a spatial object in one dataset is the same as or part of a spatial object in the other dataset. In different datasets, the same real world object is usually represented using different geometries or coordinates. Previously, we proposed a number of qualitative spatial logics (a logic of NEAR and FAR for buffered points, a logic of NEAR and FAR for buffered geometries and a logic of Part and Whole for buffered geometries) which were developed to reason about distance relations between spatial objects from different datasets, tolerating slight differences in their geometric representations [Du et al., 2013; Du and Alechina, 2016]. These spatial logics have been used to validate matching relations regarding the distance relations between spatial objects. The intuition is that two spatial objects which are definitely close in one dataset cannot be matched to two spatial objects which are definitely far away in the other dataset. However, these spatial logics do not cover the direction aspect, which is an important dimension of spatial relations. In this work, we propose a new spatial logic for validating matching relations with respect to direction relations between spatial objects. Using the relations defined in the new logic, the following intuition can be formalised: if a spatial object $a$ is definitely to the east of a spatial object $b$ in one dataset, then the spatial object corresponding to $a$ in the other dataset cannot be definitely to the west of the spatial object corresponding to $b$.

Consider the case where every spatial object is represented as a single point. We assume that the distance between every pair of corresponding points from different datasets is less than or equal to a positive real number $\sigma . \sigma$ is referred to as a margin of error. The value of $\sigma$ can be determined empirically by comparing two geospatial datasets representing the same objects, and finding the largest 'distortion' which exists between any pair of objects. With respect to a point $p$, if a point $q$ is within the bounding box of the $\sigma$-buffer of $p$ (the $\sigma$-buffer contains exactly all the points within $\sigma$ distance of $p$ ), then $q$ is considered to be too close to talk about its exact direction. We say that $q$ is not to the north, not to the south, not to the east and not to the west of $p$. In the logic of NEAR and FAR for buffered points [Du et al., 2013], two points are $N E A R$, if their distance is within $2 \sigma$; two points are $F A R$, if their distance is greater than $4 \sigma$. A gap is left between $N E A R$ and FAR so that two points are not NEAR and not FAR, if their distance is greater than $2 \sigma$ and within $4 \sigma$. Similar to the way in which the relations $N E A R$ and $F A R$ were defined, we will leave some gaps or indeterminate regions between definite directions like definitely east and definitely west. E.g. for two points $p, q$ with $x$ coordinates $x_{p}, x_{q}$, we can define the three relations definitely east, not east and not west, and definitely west, as $x_{p}-x_{q}>3 \sigma$ ( $p$ is definitely to the east of $q),-\sigma \leq x_{p}-x_{q} \leq \sigma$ ( $p$ is not to the east and not the west of $q$ ) and $x_{p}-x_{q}<-3 \sigma$ ( $p$ is definitely to the west of $q$ ) respectively. Instead of introducing a constant 3 , we introduce another parameter $\tau>1$ to represent gaps or indeterminate ranges or regions. The parameter $\tau$ is referred to as the level of indeterminacy in directions. For points $p, q$, if $x_{p}$ and $x_{q}$ are within $\tau \sigma$ distance, then the direction relation between points $p, q$ are not definitely east nor definitely west. Following this initial idea, with respect to a central point $p=(0,0)$, we divide the 2D Euclidean space into 25 totally or partially bounded regions (see Figure 1). Points in different regions have different direction relations with the central red point $p$. E.g. for any point $q$ in region $1, q$ is definitely to the north and definitely to the west of $p$. The question is how to define the 25 different direction relations formally and provide a sound and complete axiomatisation to reason with them.

Several qualitative spatial or temporal calculi have been developed for formalizing and reasoning about direction or ordering relations [Aiello et al., 2007; Ligozat, 2012]. These include the point calculus [Vilain and Kautz, 1986] which defines three ordering relations $<$ (less than), $>$ (greater than) and $e q$ (equal) for points in a 1D Euclidean space, Allen's calculus [Allen, 1983], the cardinal direction calculus (CDC) which extends the point calculus to 2D Euclidean 


\begin{tabular}{|c|c|c|c|c|}
\hline & & & & \\
\hline 1 & 2 & 3 & 4 & 5 \\
\hline 6 & 7 & 8 & 9 & 10 \\
\hline 11 & 12 & 13 & 14 & 15 \\
\hline 16 & 17 & 18 & 19 & 20 \\
\hline 21 & 22 & 23 & 24 & 25 \\
\hline
\end{tabular}

Figure 1: The 2D Euclidean space is divided into 25 totally or partially bounded regions. The red dot in region 13 is the central point $p=(0,0)$.

space [Ligozat, 1998], the rectangle algebra [Balbiani et al., 1998], the $2 n$-star calculi which generalize the cardinal direction calculus by introducing a variable $n$ referring to the granularity or the degree of refinement for defining direction relations [Renz and Mitra, 2004], and cardinal direction relations between regions [Goyal and Egenhofer, 1997; Skiadopoulos and Koubarakis, 2004; Skiadopoulos and Koubarakis, 2005]. Beside these formalisms where direction or ordering relations are defined using binary relations, there exist several spatial formalisms which define direction relations using ternary relations. These spatial formalisms include the $\mathcal{L} \mathcal{R}$ calculus [Scivos and Nebel, 2004], the flip-flop calculus [Ligozat, 1993], the double-cross calculus [Freksa, 1992], the 5-intersection calculus [Billen and Clementini, 2004], etc., where relations like left, right, after, between, before, etc. are defined.

In this paper, we propose a logic of directions for points $(L D)$ over 2D Euclidean space for defining and reasoning about the direction relations shown in Figure 1. Differing from the cardinal direction calculus, in the logic $L D$, we define direction relations with respect to the margin of error $\sigma$ for tolerating slight differences in geometric representations in different geospatial datasets/maps, and the level of indeterminacy in directions $\tau$. Over Euclidean spaces, there exist some sound and complete axiomatisations for spatial formalisms [Szczerba and Tarski, 1979; Balbiani et al., 2007; Tarski, 1959; Tarski and Givant, 1999; Trybus, 2010]; however, none of them considers direction relations. Here we provide a sound and complete axiomatisation for the spatial logic $L D$ which formalises direction relations between points. Some spatial logics, which can encode directions, are undecidable, e.g. the compass logic [Marx and Reynolds, 1999] and SpPNL [Morales et al., 2007]. The satisfiability problem of some spatial logics (e.g. Cone [Montanari et al., 2009] and SOSL [Walega and Zawidzki, 2019]) are PSPACEcomplete. Here we show that the satisfiability problem of $L D$ is NP-complete.

The logic $L D$ could be used for checking consistency of sameAs matches between two real world geospatial datasets (e.g. Ordnance Survey of Great Britain and OpenStreetMap data) regarding direction information. A sound and complete axiomatisation of $L D$ is an important and useful tool for developing an automated reasoner and performing automated axiom pinpointing [Baader and Peñaloza, 2010] for debugging matches between geospatial objects, as was done, for

\begin{tabular}{|l||l|l|l|l|l|}
\hline & $d W$ & $s W$ & $n E W$ & $s E$ & $d E$ \\
\hline \hline$d N$ & $d N d W$ & $d N s W$ & $d N n E W$ & $d N s E$ & $d N d E$ \\
$s N$ & $s N d W$ & $s N s W$ & $s N n E W$ & $s N s E$ & $s N d E$ \\
$n N S$ & $n N S d W$ & $n N S s W$ & $n N S n E W$ & $n N S s E$ & $n N S d E$ \\
$s S$ & $s S d W$ & $s S s W$ & $s S n E W$ & $s S s E$ & $s S d E$ \\
$d S$ & $d S d W$ & $d S s W$ & $d S n E W$ & $d S s E$ & $d S d E$ \\
\hline
\end{tabular}

Table 1: 25 jointly exhaustive and pairwise disjoint direction relations. Each entry in the table corresponds to the spatially corresponding entry in Figure 1, e.g. $n N S s W$ corresponds to entry 12.

example, in [Du et al., 2015] for the logic of Part and Whole for buffered geometries.

\section{A Logic of Directions For Points}

We present a logic of directions for points $(L D)$, which defines six primary direction relations: east $(E)$, west $(W)$, and indeterminate east/west $\left(I_{e w}\right)$, north $(N)$, south $(S)$ and indeterminate north/south $\left(I_{n s}\right) . L D$ is a family of logics $L D^{\tau}$ parameterised by a level of indeterminacy parameter $\tau$.

Let $A$ be a finite set of individual names. The language $L(L D, A)$ (we omit $A$ for brevity below) is defined as

$$
\begin{gathered}
\phi, \psi:=E(a, b)|W(a, b)| I_{e w}(a, b)|N(a, b)| S(a, b) \mid \\
\left|I_{n s}(a, b)\right| \neg \phi \mid \phi \wedge \psi
\end{gathered}
$$

where $a, b \in A, \phi \vee \psi={ }_{\text {def }} \neg(\neg \phi \wedge \neg \psi), \phi \rightarrow \psi={ }_{\text {def }} \neg(\phi \wedge$ $\neg \psi), \phi \leftrightarrow \psi={ }_{\text {def }}(\phi \rightarrow \psi) \wedge(\psi \rightarrow \phi), \perp==_{\text {def }} \phi \wedge \neg \phi$.

We interpret $L(L D)$ over 2D Euclidean models based on the 2D Euclidean space $\mathbb{R}^{2}$. Models of $L D^{\tau}$ are called $\tau$ models.

Definition 1 (2D Euclidean $\tau$-model of $L D^{\tau}$ ). $A$ 2D Euclidean $\tau$-model $M$ is a tuple $(\mathcal{I}, \sigma, \tau)$, where $\mathcal{I}$ is an interpretation function which maps each individual name in $A$ to an element of $\mathbb{R}^{2}, \sigma \in \mathbb{R}_{>0}$ is a margin of error, and $\tau \in \mathbb{N}_{>1}$ refers to the level of indeterminacy in directions. The notion of $M=_{L D} \phi$ (a formula $\phi$ of $L D$ is true in $\tau$-model $M$ ) is defined as follows:

$$
\begin{aligned}
& M=_{L D} E(a, b) \text { iff } x_{a}-x_{b}>\sigma ; \\
& M=_{L D} W(a, b) \text { iff } x_{a}-x_{b}<-\sigma ; \\
& M={ }_{L D} I_{e w}(a, b) \text { iff }-\tau \sigma \leq x_{a}-x_{b} \leq \tau \sigma ; \\
& M={ }_{L D} N(a, b) \text { iff } y_{a}-y_{b}>\sigma ; \\
& M={ }_{L D} S(a, b) \text { iff } y_{a}-y_{b}<-\sigma ; \\
& M={ }_{L D} I_{n s}(a, b) \text { iff }-\tau \sigma \leq y_{a}-y_{b} \leq \tau \sigma ; \\
& M={ }_{L D} \neg \phi \text { iff } M \forall{ }_{L D} \phi ; \\
& M=_{L D} \phi \wedge \psi \text { iff } M=_{L D} \phi \text { and } M=_{L D} \psi,
\end{aligned}
$$

where $a, b \in A, \mathcal{I}(a)=\left(x_{a}, y_{a}\right), \mathcal{I}(b)=\left(x_{b}, y_{b}\right), \phi, \psi$ are formulas in $L(L D)$.

$\tau$ is defined as a natural number rather than a real in order to facilitate the proof of Lemma 5. In practice, an integer $\tau$ is always likely to be sufficiently expressive.

The notions of $\tau$-validity and $\tau$-satisfiability of $L D$ formulas in 2D Euclidean $\tau$-models are standard. An $L(L D)$ formula is $\tau$-satisfiable if it is true in some 2D Euclidean $\tau$ model. An $L(L D)$ formula $\phi$ is $\tau$-valid $\left(\mid={ }_{L D}^{\tau} \phi\right)$ if it is true 
in all 2D Euclidean $\tau$-models (hence if its negation is not $\tau$ satisfiable). The logic $L D^{\tau}$ is the set of all $\tau$-valid formulas of $L(L D)$.

As shown by Lemma 1 below, $\sigma$ is a scaling factor.

Lemma 1. For every $\tau \in \mathbb{N}_{>1}, \sigma_{1}, \sigma_{2} \in \mathbb{R}_{>0}$, if an $L(L D)$ formula $\phi$ is true in a $2 D$ Euclidean $\tau$-model $M=\left(\mathcal{I}, \sigma_{1}, \tau\right)$, then it is true in a $2 D$ Euclidean $\tau$-model $M^{\prime}=\left(\mathcal{I}^{\prime}, \sigma_{2}, \tau\right)$ such that $\mathcal{I}(a)=\left(x_{a}, y_{a}\right)$ iff $\mathcal{I}^{\prime}(a)=\left(\frac{x_{a} \sigma_{2}}{\sigma_{1}}, \frac{y_{a} \sigma_{2}}{\sigma_{1}}\right)$.

The proof is by straightforward verification of truth conditions in Definition 1.

We introduce the following definitions as 'syntactic sugar'.

\section{Definition 2.}

definitely east $d E(a, b)=_{\text {def }} E(a, b) \wedge \neg I_{e w}(a, b)$

somewhat east $s E(a, b)={ }_{\operatorname{def}} E(a, b) \wedge I_{e w}(a, b)$

neither east nor west $n E W(a, b)={ }_{\text {def }} \neg E(a, b) \wedge$ $\neg W(a, b)$

somewhat west $s W(a, b)={ }_{\operatorname{def}} W(a, b) \wedge I_{e w}(a, b)$

definitely west $d W(a, b)={ }_{\operatorname{def}} W(a, b) \wedge \neg I_{e w}(a, b)$

definitely north $d N(a, b)={ }_{\operatorname{def}} N(a, b) \wedge \neg I_{n s}(a, b)$

somewhat north $s N(a, b)={ }_{\operatorname{def}} N(a, b) \wedge I_{n s}(a, b)$

neither north nor south $n N S(a, b)={ }_{\text {def }} \neg N(a, b) \wedge$ $\neg S(a, b)$

somewhat south $s S(a, b)={ }_{\operatorname{def}} S(a, b) \wedge I_{n s}(a, b)$

definitely south $d S(a, b)={ }_{\operatorname{def}} S(a, b) \wedge \neg I_{n s}(a, b)$

The definitions of definite or somewhat direction relations have $\tau \in \mathbb{N}_{>1}$ as a parameter. By Definitions 1 and $2, M \models_{L D} d E(a, b)$ iff $\left(x_{a}-x_{b}\right) \in(\tau \sigma, \infty) ; M \models_{L D}$ $s E(a, b)$ iff $\left(x_{a}-x_{b}\right) \in(\sigma, \tau \sigma]$. Let us call $(\tau \sigma, \infty)$ the range of $d E(a, b),(\sigma, \tau \sigma]$ the range of $s E(a, b)$. As $\tau$ decreases, the range of $d E(a, b)$ becomes wider, the range of $s E(a, b)$ becomes narrower. If $\tau$ is allowed to be 1 , then $d E(a, b) \equiv E(a, b)$ and $s E(a, b) \equiv \perp$. $\tau$ plays a similar role in defining other definite or somewhat direction relations.

There exist $5 \times 5=25$ jointly exhaustive and pairwise disjoint relations, which can be defined using the primary relations in the logic $L D$. The 25 direction relations are shown in Table 1. Each of them is defined as a conjunction of one of the relations $d W, s W, n E W, s E, d E$ and one of the relations $d N, s N, n N S, s S, d S$. These 25 direction relations correspond to the 25 regions shown in Figure 1. For instance, with respect to the central point $p$, for any point $q$ in region 2 , we have $d N s W(q, p)$ ( $q$ is definitely to the north and somewhat to the west of $p$ ).

Similar to the logic $L D$, we could define a logic over 3D or higher Euclidean space. If we only use east and west (or north and south), we get a logic $L D 1$ over 1D Euclidean space. The soundness, completeness, decidability and complexity results can be obtained similarly. The point calculus and the Cardinal Direction Calculus can be seen as a special case of $L D 1$ and $L D$ respectively, if $\sigma$ is allowed to be 0 . Finally, we observe that there exist different (from $L D$ ) extensions of the point calculus and Allen's calculus, for example, introducing the concept of granularity [Cohen-Solal et al., 2015]; a granularity is defined as a sequence of sets of time points where the natural order of the time points are preserved.

\section{A Complete Axiomatisation for LD}

Here we will first describe some results for systems of linear inequalities that are used later in the proofs. Then for each level of indeterminacy $\tau$, we present an axiomatisation (a set of axioms) of $L D^{\tau}$, and prove soundness and completeness of the axiomatisation.

\subsection{Deciding Linear Inequalities by Computing Loop Residues}

We recap the definitions from [Shostak, 1981]. Let $S$ be a set of linear inequalities of the form $a x+b y \leq c$, where $x, y$ are real variables and $a, b, c$ are reals. Without loss of generality, we assume one of the variables in $S$, denoted as $v_{0}$, is special, appearing only with coefficient zero. It is called the 'zero variable'. All other variables in $S$ have nonzero coefficients.

The graph for $S$, denoted as $G$, is constructed as follows. $G$ contains a vertex for each variable in $S$ and an edge for each inequality, where each vertex is labelled with its associated variable and each edge is labelled with its associated inequality. For example, the edge labelled with $a x+b y \leq c$ connects the vertex labelled with $x$ and the vertex labelled with $y$.

Let $P$ be a path through $G$, given by a sequence $v_{1}, \ldots, v_{n+1}$ of vertices and a sequence $e_{1}, \ldots, e_{n}$ of edges, $n \geq 1$. The triple sequence for $P$ is

$$
\left(a_{1}, b_{1}, c_{1}\right),\left(a_{2}, b_{2}, c_{2}\right), \ldots,\left(a_{n}, b_{n}, c_{n}\right)
$$

where for each $i \in[1, n], a_{i} v_{i}+b_{i} v_{i+1} \leq c_{i}$ is the inequality labelling $e_{i}$. A path is a loop if its first and last vertices are the same. A loop is simple if its intermediate vertices are distinct. $P$ is admissible if for $i \in[1, n-1], b_{i}$ and $a_{i+1}$ have opposite signs (one is strictly positive and the other is strictly negative). Definitions and results that follow apply to admissible paths.

The residue inequality of an admissible path $P$ is defined as the inequality obtained from $P$ by applying transitivity to the inequalities labelling its edges. The residue $r_{p}$ of $P$ is defined as the triple $\left(a_{p}, b_{p}, c_{p}\right)$,

$$
\left(a_{p}, b_{p}, c_{p}\right)=\left(a_{1}, b_{1}, c_{1}\right) *\left(a_{2}, b_{2}, c_{2}\right) * \cdots *\left(a_{n}, b_{n}, c_{n}\right)
$$

where $\left(a_{1}, b_{1}, c_{1}\right), \ldots,\left(a_{n}, b_{n}, c_{n}\right)$ is the triple sequence for $P$ and $*$ is the binary operation on triples defined by

$$
(a, b, c) *\left(a^{\prime}, b^{\prime}, c^{\prime}\right)=\left(k a a^{\prime},-k b b^{\prime}, k\left(c a^{\prime}-c^{\prime} b\right)\right)
$$

where $k=a^{\prime} /\left|a^{\prime}\right|$. The residue inequality of $P$ is $a_{P} x+$ $b_{P} y \leq c_{P}$, where $x, y$ are the first and last vertices of $P$.

Lemma 2. [Shostak, 1981] Any point (i.e. assignment of reals to variables) that satisfies the inequalities labelling on admissible path $P$ also satisfies the residue inequality of $P$.

Let $P$ be an admissible loop with initial vertex $x$. By Lemma 2, any point satisfying the inequalities along $P$ also satisfies $a_{P} x+b_{P} x \leq c_{P}$. If $a_{P}+b_{P}=0$ and $c_{p}<0$, then the residue inequality of $P$ is false, and $P$ is called an infeasible loop.

Let $G$ be the graph for $S$. A closure $G^{\prime}$ of $G$ is obtained by adding, for each simple admissible loop $P$ (modulo permutation and reversal) of $G$, a new edge labelled with the residue inequality of $P$. A graph is closed if it is a closure of itself. 
Theorem 1. [Shostak, 1981] Let $S$ be a set of linear inequalities of the form $a x+b y \leq c$, where $x$, $y$ are real variables and $a, b, c$ are real number constants; let $G$ be a closed graph for $S$. Then $S$ is satisfiable iff $G$ has no simple infeasible loop.

Theorem 1 is about inequalities of the form $a x+b y \leq c$ only. It was extended to include both strict and non-strict inequalities [Shostak, 1981]. We say an admissible path is strict if one or more of its edges is labelled with a strict inequality, i.e. an inequality of the form $a x+b y<c$. Then a strict admissible loop $P$ with residue $\left(a_{P}, b_{P}, c_{P}\right)$ is infeasible, if $a_{P}+b_{P}=0$ and $c_{P} \leq 0$. Corollary 1 is stated for the case where inequalities are of the form $x-y \leq c$ or $x-y<c$. Lemma 3 is provided to help readers understand Corollary 1. It follows from the definition of closed graph.

Lemma 3. [Shostak, 1981] Let $S$ be a set of linear inequalities of the form $x-y \leq c$ or $x-y<c$, where $x$, $y$ are real variables and $c$ is a real number constant. Then the graph for $S$ is closed.

Corollary 1. [Litvintchouk and Pratt, 1977; Pratt, 1977; Shostak, 1981] Let $S$ be a set of linear inequalities of the form $x-y \leq c$ or $x-y<c$, where $x, y$ are real variables and $c$ is a real number constant; $G$ be a graph for $S$. The set $S$ is not satisfiable iff $G$ has a simple infeasible loop.

\subsection{Axiomatising LD}

The calculus below (which we will also refer to as $L D^{\tau}$ ) is sound and complete for $L D^{\tau}$ (for any $\tau$ ). Here, $a$ and $b$ are meta variables which may be instantiated by any individual name. There are 13 axiom schemas (AS 0 to AS 12) and one inference rule.

AS 0 All tautologies of classical propositional logic

AS $1 \neg W(a, a)$;

AS $2 E(a, b) \leftrightarrow W(b, a)$;

AS $3 I_{e w}(a, b) \rightarrow I_{e w}(b, a)$;

AS $4 I_{e w}(a, b) \leftrightarrow(\neg d E(a, b) \wedge \neg d W(a, b))$;

AS 5 For any $n \in \mathbb{N}_{>1}$ :

$R_{1}\left(a_{0}, a_{1}\right) \wedge \cdots \wedge R_{n}\left(a_{n-1}, a_{0}\right) \rightarrow \perp$, where for every $i$ such that $1 \leq i \leq n, R_{i} \in\{W, d W, \neg E, \neg d E\}$, and number $(W)+\tau *$ number $(d W)=$ number $(\neg E)+$ $\tau *$ number $(\neg d E)$;

AS 6 For any $n \in \mathbb{N}_{>0}$ :

$R_{1}\left(a_{0}, a_{1}\right) \wedge \cdots \wedge R_{n}\left(a_{n-1}, a_{n}\right) \rightarrow W\left(a_{0}, a_{n}\right)$,

where for every $i$ such that $1 \leq i \leq n$, $R_{i} \in\{W, d W, \neg E, \neg d E\}$, and number $(W)+\tau *$ number $(d W)>$ number $(\neg E)+\tau *$ number $(\neg d E)$;

AS $7 \neg S(a, a)$;

AS $8 N(a, b) \leftrightarrow S(b, a)$;

AS $9 I_{n s}(a, b) \rightarrow I_{n s}(b, a)$;

AS $10 I_{n s}(a, b) \leftrightarrow(\neg d N(a, b) \wedge \neg d S(a, b))$;

AS 11 For any $n \in \mathbb{N}_{>1}$ :

$R_{1}\left(a_{0}, a_{1}\right) \wedge \cdots \wedge R_{n}\left(a_{n-1}, a_{0}\right) \rightarrow \perp$, where for every $i$ such that $1 \leq i \leq n, R_{i} \in\{S, d S, \neg N, \neg d N\}$, and number $(S)+\tau *$ number $(d S)=$ number $(\neg N)+\tau *$ number $(\neg d N)$;
AS 12 For any $n \in \mathbb{N}_{>0}$ :

$R_{1}\left(a_{0}, a_{1}\right) \wedge \cdots \wedge R_{n}\left(a_{n-1}, a_{n}\right) \rightarrow S\left(a_{0}, a_{n}\right)$, where for every $i$ such that $1 \leq i \leq n$, $R_{i} \in\{S, d S, \neg N, \neg d N\}$, and number $(S)+\tau *$ number $(d S)>$ number $(\neg N)+\tau *$ number $(\neg d N)$;

MP Modus ponens: $\phi, \phi \rightarrow \psi \vdash \psi$.

In AS 5, 6, 11 and 12, $n$ is the number of conjuncts in the antecedent of an axiom, number $(\alpha)$ denotes the number of occurrences of $\alpha$ in $R_{1}, \ldots, R_{n}$. In AS 5 and AS 11, n> 1 because at least two conjuncts are required to make an equality like number $(W)+\tau *$ number $(d W)=$ number $(\neg E)+\tau *$ number $(\neg d E)$ true. For AS 5, suppose that $n=4$, number $(W)$, number $(d W), \quad$ number $(\neg E)$ and number $(\neg d E)$ are all equal to 1 , then an axiom satisfying this is $W\left(a_{0}, a_{1}\right) \wedge \neg d E\left(a_{1}, a_{2}\right) \wedge \neg E\left(a_{2}, a_{3}\right) \wedge d W\left(a_{3}, a_{0}\right) \rightarrow$ $\perp$ (the order of the appearance of $W, d W, \neg E, \neg d E$ does not matter).

The notion of $\tau$-derivability $\Gamma \vdash_{L D}^{\tau} \phi$ in the $L D^{\tau}$ calculus is standard. An $L(L D)$ formula $\phi$ is $\tau$-derivable if $\vdash_{L D}^{\tau} \phi$; a set of $L(L D)$ formulas. $\Gamma$ is $\tau$-inconsistent if for some formula $\phi$ it $\tau$-derives both $\phi$ and $\neg \phi$ (otherwise it is $\tau$-consistent).

Theorem 2. For every $\tau \in \mathbb{N}_{>1}$, the $L D^{\tau}$ calculus is sound and complete for $2 D$ Euclidean $\tau$-models, i.e. $\vdash_{L D}^{\tau} \phi \Leftrightarrow$ $\models{ }_{L D}^{\tau} \phi$ (every $\tau$-derivable formula is $\tau$-valid and every $\tau$ valid formula is $\tau$-derivable).

For every $\tau \in \mathbb{N}_{>1}$, the proof of soundness (every $L D \tau$ derivable formula is $\tau$-valid) is by an easy induction on the length of the derivation of $\phi$. By truth definitions of the direction relations (Definition 1), AS 1-12 are valid and modus ponens preserves validity.

In the rest of this section, we prove completeness. We will actually prove that for every $\tau \in \mathbb{N}_{>1}$, if a finite set of $L(L D)$ formulas $\Sigma$ is $\tau$-consistent, then there is a $2 D$ Euclidean $\tau$ model satisfying it. Any finite set of formulas $\Sigma$ can be rewritten as a formula $\psi$ that is the conjunction of all the formulas in $\Sigma$. $\Sigma$ is $\tau$-consistent iff $\psi$ is $\tau$-consistent $\left(\nvdash_{L D}^{\tau} \neg \psi\right)$. If there is a 2D Euclidean $\tau$-model $M$ satisfying $\Sigma$, then $M$ satisfies $\psi$, hence $\nvdash_{L D}^{\tau} \neg \psi$. Therefore, by showing that 'if $\Sigma$ is $\tau$-consistent, then there exists a 2D Euclidean $\tau$-model satisfying it', we show that 'if $\forall_{L D}^{\tau} \neg \psi$, then $\forall_{L D}^{\tau} \neg \psi$ '. This shows that $\forall_{L D}^{\tau} \phi \Rightarrow \nvdash_{L D}^{\tau} \phi$ and by contraposition we get completeness.

First, we will show that the truth conditions of any set of $L(L D)$ formulas can be expressed as a set of inequalities of the form $x_{1}-x_{2} \leq c$ or $x_{1}-x_{2}<c$.

Lemma 4. An $L(L D)$ formula of the form $(\neg) E(a, b)$, $(\neg) W(a, b), \quad(\neg) d E(a, b), \quad(\neg) d W(a, b), \quad(\neg) N(a, b)$, $(\neg) S(a, b),(\neg) d N(a, b),(\neg) d S(a, b)$ is $\tau$-satisfiable iff an expression of the form $x_{1}-x_{2} \leq c$ or $x_{1}-x_{2}<c$ is satisfiable.

Proof. Definition 3 shows how to translate such formulas to corresponding inequalities. The translation can be easily verified to correspond to the truth definitions in Definition 1. 
Definition 3 ( $\tau$ - $\sigma$-translation). The ' $\tau$ - $\sigma$-translation' function $\operatorname{tr}(\tau, \sigma)$ is defined as follows:

$$
\begin{aligned}
& \operatorname{tr}(\tau, \sigma)(E(a, b))=\left(x_{b}-x_{a}<-\sigma\right) ; \\
& \operatorname{tr}(\tau, \sigma)(W(a, b))=\left(x_{a}-x_{b}<-\sigma\right) ; \\
& \operatorname{tr}(\tau, \sigma)(d E(a, b))=\left(x_{b}-x_{a}<-\tau \sigma\right) ; \\
& \operatorname{tr}(\tau, \sigma)(d W(a, b))=\left(x_{a}-x_{b}<-\tau \sigma\right) ; \\
& \operatorname{tr}(\tau, \sigma)(N(a, b))=\left(y_{b}-y_{a}<-\sigma\right) ; \\
& \operatorname{tr}(\tau, \sigma)(S(a, b))=\left(y_{a}-y_{b}<-\sigma\right) ; \\
& \operatorname{tr}(\tau, \sigma)(d N(a, b))=\left(y_{b}-y_{a}<-\tau \sigma\right) ; \\
& \operatorname{tr}(\tau, \sigma)(d S(a, b))=\left(y_{a}-y_{b}<-\tau \sigma\right) ; \\
& \operatorname{tr}(\tau, \sigma)(\neg \phi)=\neg(\operatorname{tr}(\phi)), \text { where } \neg\left(z_{1}-z_{2}<c\right)=\left(z_{2}-\right. \\
& \left.\quad z_{1} \leq-c\right) .
\end{aligned}
$$

The completeness theorem below is proven by rewriting a consistent $L(L D)$ formula $\phi$ into disjunctive normal form, where each disjunct $\phi_{i}$ is $\tau$-satisfiable, iff a set of linear inequalities $S_{i}$ is satisfiable, iff the graphs of $S_{i}$ have no simple infeasible loop (Corollary 1 of Theorem 1 ). We proceed by contradiction, supposing every such graph has a simple infeasible loop $P$. From $P$ we can obtain $L(L D)$ formulas as conjuncts in $\phi_{i}$. Applying the axioms, we show $\perp$ is $\tau$ derivable from every $\phi_{i}$, thus $\perp$ is $\tau$-derivable from $\phi$, which contradicts that $\phi$ is $\tau$-consistent.

Theorem 3. For every $\tau \in \mathbb{N}_{>1}$, if a finite set of $L(L D)$ formulas $\Sigma$ is $\tau$-consistent, then there is a $2 D$ Euclidean $\tau$ model satisfying it.

Proof. Take an arbitrary $\tau \in \mathbb{N}_{>1}$. Suppose a finite set of $L(L D)$ formulas $\Sigma$ is $\tau$-consistent. We obtain $\Sigma^{\prime}$ by rewriting every $I_{e w}(a, b)$ in $\Sigma$ as $\neg d E(a, b) \wedge \neg d W(a, b)$, every $I_{n s}(a, b)$ in $\Sigma$ as $\neg d N(a, b) \wedge \neg d S(a, b)$. By AS 4 and AS $10, \Sigma$ and $\Sigma^{\prime}$ are logically equivalent. $\Sigma^{\prime}$ can be rewritten as a formula $\phi$ that is the conjunction of all the formulas in $\Sigma^{\prime}$. We rewrite the $L(L D)$ formula $\phi$ into disjunctive normal form $\phi_{1} \vee \cdots \vee \phi_{n}(n>0)$. Then every literal is of one of the forms $E(a, b), W(a, b), d E(a, b), d W(a, b), N(a, b)$, $S(a, b), d N(a, b), d S(a, b)$, or their negations. Then $\phi$ is satisfiable in a 2D Euclidean $\tau$-model, iff at least one of its disjuncts $\phi_{i}$ is $\tau$-satisfiable. We obtain a set of inequalities $S_{i}$ by translating every literal in a disjunct $\phi_{i}$ as in Definition 3 . Then the inequalities in $S_{i}$ are of the form $x_{a}-x_{b}<c$, $x_{a}-x_{b} \leq c, y_{a}-y_{b}<c$ or $y_{a}-y_{b} \leq c$, where $x_{a}, x_{b}, y_{a}, y_{b}$ are real variables and $c$ is a real constant. We call variables like $x_{a}, x_{b} x$ variables and variables like $y_{a}, y_{b} y$ variables. Divide $S_{i}$ into two sets $S_{i}^{x}$ and $S_{i}^{y}$, such that $S_{i}^{x}$ and $S_{i}^{y}$ contain all the inequalities involving $x$ variables and $y$ variables respectively. By Corollary 1 of Theorem $1, \phi_{i}$ is $\tau$-satisfiable iff the graph $G_{i}^{x}$ of $S_{i}^{x}$ has no simple infeasible loop and the graph $G_{i}^{y}$ of $S_{i}^{y}$ has no simple infeasible loop. To show there is a 2D Euclidean $\tau$-model satisfying $\Sigma$, it is sufficient to show there exists a disjunct $\phi_{i}$ such that the graph $G_{i}^{x}$ of $S_{i}^{x}$ has no simple infeasible loop and the graph $G_{i}^{y}$ of $S_{i}^{y}$ has no simple infeasible loop.

We prove this by contradiction. Suppose for every disjunct $\phi_{i}$, the graph $G_{i}^{x}$ of $S_{i}^{x}$ has a simple infeasible loop (Case 1) or the graph $G_{i}^{y}$ of $S_{i}^{y}$ has a simple infeasible loop (Case 2). We present the proof for Case 1. Case 2 is similar.
If $G_{i}^{x}$ has a simple infeasible loop $P$, then $P$ is either strict or non-strict. Let $m$ denote the sum of the constants $c$ around the loop $P$. Based on the definition of infeasible loop, if $P$ is strict, then $m \leq 0$; otherwise, $m<0$. By Definition 3, if a strict inequality $x_{a}-x_{b}<c$ is in $S_{i}^{x}$, then $c$ is equal to $-\sigma$ or $-\tau \sigma$; if a non-strict inequality $x_{a}-x_{b} \leq c$ is in $S_{i}^{x}$, then $c$ is equal to $\sigma$ or $\tau \sigma$, where $\tau, \sigma$ are positive numbers (hence $c>0$ ). If $P$ is non-strict, then all the inequalities in it are of the form $x_{a}-x_{b} \leq c$ where $c>0$ and the sum of such $c$ is positive. This contradicts the fact that $m<0$ for non-strict infeasible loops. Therefore $P$ is strict, hence $m \leq 0$. We consider the two cases where $m=0$ and $m<0$ separately.

1. If $m=0$, then the sum of the constants around the loop $P$ is equal to 0 . Without loss of generality, let us assume $P$ consists of vertices $x a_{0}, x a_{1}, \ldots, x a_{n-1}, x a_{0}$. Since $P$ is admissible, the linear inequalities in $P$ are of the form $\left(x a_{0}-x a_{1}\right) ? c_{1}, \ldots,\left(x a_{n-1}-x a_{0}\right) ? c_{n}$, where $?$ is $\leq$ or $<$, and for every $i$ such that $1 \leq i \leq n, c_{i}$ is $\sigma,-\sigma, \tau \sigma$ or $-\tau \sigma$. Then we translate the linear inequalities in $P$ to formulas as follows. We translate every linear inequality of the form $x_{a}-x_{b}<-\sigma$ to $W(a, b)$; every $x_{a}-x_{b}<-\tau \sigma$ to $d W(a, b)$; every $x_{a}-x_{b} \leq \sigma$ to $\neg E(a, b)$; every $x_{a}-x_{b} \leq \tau \sigma$ to $\neg d E(a, b)$. In this way, from $P$ we obtain a sequence of formulas of the form $R_{1}\left(a_{0}, a_{1}\right), \ldots, R_{n}\left(a_{n-1}, a_{0}\right)$, where for every $i$ such that $1 \leq i \leq n, R_{i} \in\{W, d W, \neg E, \neg d E\}$. Since the sum of the constants around $P$ is equal to 0 , number $(W)+\tau *$ number $(d W)=$ number $(\neg E)+\tau *$ number $(\neg d E)$ and $n \geq 2$. By AS $5, R_{1}\left(a_{0}, a_{1}\right) \wedge \ldots \wedge$ $R_{n}\left(a_{n-1}, a_{0}\right) \rightarrow \perp$. By Definition 3 , for every occurrence of $W(a, b)$ in $R_{1}\left(a_{0}, a_{1}\right) \wedge \ldots \wedge R_{n}\left(a_{n-1}, a_{0}\right)$, it or $E(b, a)$ is a conjunct in $\phi_{i}$; similarly, for every occurrence of $d W(a, b)$, it or $d E(b, a)$ is a conjunct in $\phi_{i}$; for every occurrence of $\neg E(a, b)$, it or $\neg W(b, a)$ is a conjunct in $\phi_{i}$; for every occurrence of $\neg d E(a, b)$, it or $\neg d W(b, a)$ is a conjunct in $\phi_{i}$. By AS 2, $W(a, b) \leftrightarrow E(b, a)$. By Definition 2, AS 2 and AS 3, $d W(a, b) \leftrightarrow d E(b, a)$. Therefore, $\perp$ is $\tau$-derivable from $\phi_{i}$.

2. If $m<0$, then the sum of the constants around the loop $P$ is negative. In the same way described above, from $P$ we obtain a sequence of formulas of the form $R_{1}\left(a_{0}, a_{1}\right), \ldots, R_{n}\left(a_{n-1}, a_{0}\right)$, where for every $i$ such that $1 \leq i \leq n, R_{i} \in\{W, d W, \neg E, \neg d E\}$. Since the sum of the constants around the loop $P$ is negative, number $(W)+$ $\tau *$ number $(d W)>$ number $(\neg E)+\tau *$ number $(\neg d E)$ and $n \geq 1$. By AS $6, R_{1}\left(a_{0}, a_{1}\right) \wedge \ldots \wedge R_{n}\left(a_{n-1}, a_{0}\right) \rightarrow$ $W\left(a_{0}, a_{0}\right)$. By AS $1, W\left(a_{0}, a_{0}\right) \rightarrow \perp$. Following the same argument above, $\perp$ is $\tau$-derivable from $\phi_{i}$.

In each case, $\perp$ is $\tau$-derivable from $\phi_{i}$. Thus every disjunct $\phi_{i}$ is not $\tau$-consistent, hence $\phi$ is not $\tau$-consistent. This contradicts the fact that $\Sigma$ is $\tau$-consistent.

\section{Decidability and Complexity of LD}

We show that for every $\tau \in \mathbb{N}_{>1}$, the satisfiability problem for $L D^{\tau}$ is NP-complete.

Lemma 5. For every $\tau \in \mathbb{N}_{>1}$, let $S$ be a set of linear inequalities obtained by applying the ' $\tau$ - $\sigma$-translation' function over $L(L D)$ formulas as shown in Definition 3, where $\sigma=1$; $n$ be the number of variables in $S, n>0$. If $S$ is satisfiable, then it has a solution where for every variable, a rational 
number $t \in[-n \tau, n \tau]$ is assigned to it and the binary representation size of $t$ is polynomial in $n$ and $\tau$.

Proof. Take an arbitrary $\tau \in \mathbb{N}_{>1}$. By Definition 3, every linear inequality in $S$ is of the form $x_{1}-x_{2} \leq c$ or $x_{1}-x_{2}<c$, where $x_{1}, x_{2}$ are real variables and $c$ is a real number constant. Let $G$ be a graph for $S$. By Corollary $1, S$ is satisfiable iff $G$ has no simple infeasible loop. The construction of a solution of $S$ is by extending the proof of Theorem 1 [Shostak, 1981] (pp. 777 and 778), which is for non-strict inequalities only, to include both strict and non-strict inequalities. If $G$ has no simple infeasible loop, a solution of $S$ can be constructed as follows. Let $v_{1}, \ldots, v_{n-1}$ be the variables of $S$ other than $v_{0}$ (the zero variable). We construct a sequence $\hat{v}_{0}, \hat{v}_{1}, \ldots, \hat{v}_{n-1}$ of reals (a solution of $S$ ) and a sequence $G_{0}, G_{1}, \ldots, G_{n-1}$ of graphs inductively:

1. Let $\hat{v}_{0}=0$ and $G_{0}=G$.

2. If $\hat{v}_{i}$ and $G_{i}$ have been determined for $0 \leq i<j<n$, let

$$
\begin{aligned}
& \sup _{j}=\min \left\{\frac{c_{P}}{a_{P}} \mid P \text { is an admissible path from } v_{j} \text { to } v_{0}\right. \text { in } \\
& \left.\quad G_{j-1} \text { and } a_{P}>0\right\} \\
& \inf _{j}=\max \left\{\frac{c_{P}}{b_{P}} \mid P \text { is an admissible path from } v_{0} \text { to } v_{j}\right. \text { in } \\
& \left.\quad G_{j-1} \text { and } b_{P}<0\right\}
\end{aligned}
$$

where $\min \emptyset=\infty$ and $\max \emptyset=-\infty$. The range of $\hat{v}_{j}$ is obtained as follows.

- If there is an admissible path $P$ from $v_{j}$ to $v_{0}$ in $G_{j-1}$ such that the residue inequality of $P$ is $a_{P} v_{j}<c_{P}$, where $a_{P}>0$, and $\frac{c_{P}}{a_{P}}=\sup _{j}$, then $\hat{v}_{j}<\sup _{j}$, otherwise, $\hat{v}_{j} \leq \sup _{j}$.

- If there is an admissible path $P$ from $v_{0}$ to $v_{j}$ in $G_{j-1}$ such that the residue inequality of $P$ is $b_{P} v_{j}<c_{P}$, where $b_{P}<0$, and $\frac{c_{P}}{b_{P}}=\inf _{j}$, then $\hat{v}_{j}>\inf _{j}$, otherwise, $\hat{v}_{j} \geq \inf _{j}$.

Instead of letting $\hat{v}_{j}$ be any real number in the range [Shostak, 1981], we assign a value to $\hat{v}_{j}$ thus:

- if there exists an integer within the range of $\hat{v}_{j}$, we assign an integer to $\hat{v}_{j}$;

- otherwise, the range of $\hat{v}_{j}$ is of the form $\inf _{j}<\hat{v}_{j}<$ $\sup _{j}$. Let $\hat{v}_{j}=\frac{\inf _{j}+\sup _{j}}{2}$.

Let $G_{j}$ be obtained from $G_{j-1}$ by adding two new edges from $v_{j}$ to $v_{0}$, labelled $v_{j} \leq \hat{v}_{j}$ and $v_{j} \geq \hat{v}_{j}$ respectively.

To ensure that $\hat{v}_{j}$ and $G_{j}$ are well defined, we need the following two claims:

1 . For $1 \leq j<n$, the range of $\hat{v}_{j}$ is not empty.

2. For $0 \leq j<n, G_{j}$ has no simple infeasible loop.

We prove them by induction on $j$, similar to the proof presented in [Shostak, 1981].

Base case $j=0.1$ holds vacuously; 2 holds since $G_{0}=G$. Inductive step Suppose the claim holds for $j-1,0 \leq j-1<$ $n-1$. We will show the claim holds for $j$.

For 1 , suppose, to the contrary, that the range of $\hat{v}_{i}$ is empty. Then in $G_{j-1}$, there exist an admissible path $P_{1}$ from $v_{j}$ to $v_{0}$, where $a_{P}>0$, and an admissible path $P_{2}$ from $v_{0}$ to $v_{j}$, where $b_{P}<0$. $P_{1}$ and $P_{2}$ forms an admissible loop. By the construction of the range of $\hat{v}_{i}$ described above, if this range is empty, then the admissible loop formed by $P_{1}$ and
$P_{2}$ is infeasible, which contradicts the inductive hypothesis that $G_{j-1}$ has no simple infeasible loop.

For 2 , suppose $G_{j}$ has a simple infeasible loop $P$. Since $G_{j-1}$ has no such loop, and the loop formed by the two new edges added to $G_{j-1}$ to obtain $G_{j}$ is not infeasible, then $P$ (or its reverse) is of the form $P^{\prime} E$, where $E$ is one of the two new edges (say the one labelled $v_{j} \leq \hat{v}_{j}$; the other case is handled similarly), and $P^{\prime}$ is a path from $v_{0}$ to $v_{j}$ in $G_{j-1}$. Since $P$ is infeasible, if $P^{\prime}$ is strict, $\hat{v}_{j} \leq \frac{c_{P^{\prime}}}{b_{P^{\prime}}}$, this contradicts that $\hat{v}_{j}>\inf _{j}$, since $\inf _{j} \geq \frac{c_{P^{\prime}}}{b_{P^{\prime}}}$; if $P^{\prime}$ is not strict, $\hat{v}_{j}<\frac{c_{P^{\prime}}}{b_{P^{\prime}}}$, this contradicts that $\hat{v}_{j} \geq \inf _{j}$, since $\inf _{j} \geq \frac{c_{P^{\prime}}}{b_{P^{\prime}}}$. Q.E.D.

Now it remains to show that $\hat{v}_{j}$ satisfies $S$. Let $a x+b y \leq c$ be an inequality in $S$. We will show that $a \hat{x}+b \hat{y} \leq c$. We present the case where $a>0$ and $b<0$. The other cases are similar. Let $E$ be the edge labelled $a x+b y \leq c$ in $G_{n-1}$. Then, where $E_{1}$ is the edge labelled $\hat{x} \leq x$ in $G_{n-1}$ and $E_{2}$ is the one labelled $y \leq \hat{y}, E_{1} E E_{2}$ forms an admissible loop. Since $G_{n-1}$ has no infeasible loop, $E_{1} E E_{2}$ is feasible. Hence we have $a \hat{x}+b \hat{y} \leq c$. The proof for inequalities of the form $a x+b y<c$ is similar.

By Definition 3, $-n \tau \leq c_{P} \leq n \tau, a_{P}=1$ for $\sup _{j}, b_{P}=$ -1 for $\inf _{j}$. Therefore, $\sup _{j} \leq n \tau$, inf $_{j} \geq-n \tau$. Hence every $\hat{v}_{j}(0 \leq j<n)$ is a rational number in $[-n \tau, n \tau]$.

Now we will show that the representation size of $\hat{v}_{j}(0 \leq$ $j<n$ ) is polynomial in the size of $n$ and $\tau$. By the construction described above, $\hat{v}_{j}$ is either an integer in $[-n \tau, n \tau]$ or obtained by applying the 'average operation' $\hat{v}_{j}=\frac{\inf _{j}+\sup _{j}}{2}$. Since $\tau$ is a natural number and $\sigma=1, \inf _{1}$ and $\sup _{1}$ are integers in $[-n \tau, n \tau]$. Also, since $0<j<n$, the number of 'average operations' applied to obtain a $\hat{v}_{j}$ is at most $n$. Hence the largest denominator of the values of $\hat{v}_{j}$ is $2^{n}$. Therefore, $\hat{v}_{j}$ can be represented in a binary notation (bits) of size $\log \left(2 n \tau * 2^{n}\right)$, which is in $O(n+\log \tau)$. Hence the representation size of $\hat{v}_{j}$ is polynomial in $n$ and $\tau$.

Definition 4. Let $\phi$ be an $L(L D)$ formula. Its size $s(\phi)$ is defined as follows:

- $s(R(a, b))=3$, where $R \in\left\{E, W, I_{\text {ew }}, N, S, I_{n s}\right\}$;

- $s(\neg \phi)=1+s(\phi)$;

- $s(\phi \wedge \psi)=1+s(\phi)+s(\psi)$,

where $a, b \in A, \phi, \psi$ are formulas in $L(L D)$.

The combined size of $L(L D)$ formulas in a set $S$ is defined as the size of the conjunction of all formulas in $S$.

Theorem 4. For every $\tau \in \mathbb{N}_{>1}$, the satisfiability problem for a finite set of $L(L D)$ formulas in a $2 D$ Euclidean $\tau$-model is NP-complete.

Proof. Take an arbitrary $\tau \in \mathbb{N}_{>1}$. NP-hardness is from propositional logic being included in $L D^{\tau}$. To prove that the satisfiability problem for each $L D^{\tau}$ is in NP, we show that if a finite set of $L(L D)$ formulas $\Sigma$ is $\tau$-satisfiable, then we can guess a 2D Euclidean $\tau$-model for $\Sigma$ and verify that this model satisfies $\Sigma$, both in time polynomial in the combined size of formulas in $\Sigma$ and $\tau$. Let $s$ and $n$ denote the combined size of formulas in $\Sigma$ and the number of individual names in $\Sigma$ respectively. By Definition $4, n<s$. As $\sigma$ is a scaling 
factor, if $\Sigma$ is $\tau$-satisfiable, it is $\tau$-satisfiable in a model where $\sigma=1$.

Following the proof of Theorem 3 (first paragraph), $\Sigma$ is satisfiable in a 2D Euclidean $\tau$-model, iff there exists an $S_{i}$ such that its subsets $S_{i}^{x}$ and $S_{i}^{y}$ are both satisfiable, where $S_{i}^{x}$ and $S_{i}^{y}$ are sets of linear inequalities obtained by applying the $\tau$ - $\sigma$-translation function over $L(L D)$ formulas as shown in Definition 3. By Lemma 5, if $S_{i}^{x}$ is satisfiable, then it has a solution where for every variable, a rational number $t \in$ $[-n \tau, n \tau]$ is assigned to it and the representation size of $t$ is in $O(n+\log \tau)$ (polynomial in $n$ and $\tau$ ). The same holds for $S_{i}^{y}$. Hence for every individual name in $\Sigma$, we can guess such a pair of rational numbers for it in $O(n+\log \tau)$. Thus we can guess a 2D Euclidean $\tau$-model $M$ for $\Sigma$ in $O\left(n^{2}+n \log \tau\right)$, in time polynomial in $n$ and $\tau$. To verify that $M$ satisfies $\Sigma$, we need to check every formula in $\Sigma$. For any $R(a, b)$, where $R \in\left\{E, W, I_{e w}, N, S, I_{n s}\right\}, a, b \in A$, checking that $R(a, b)$ is true in $M$ takes $O(n+\log \tau)$ time by Definition 1 and applying bit operations. Hense, checking all formulas in $\Sigma$ takes time polynomial in $s$ and $\tau$.

An alternative decidability/membership of NP proof could use reduction to a finite set of disjunctive linear relations (DLRs) [Jonsson and Bäckström, 1998] or a $Q_{\text {basic }}$ formula [Kreutzmann and Wolter, 2014].

\section{Conclusion and Future Work}

We have introduced a new qualitative logic of directions $L D$ for reasoning about directions in $2 \mathrm{D}$ Euclidean space. We have shown it to be sound and complete, and that its decidability is NP-complete. The logic incorporates a margin of error and a level of indeterminacy in directions, that allow it to be used to compare and reason about not perfectly aligned representations of the same spatial objects in different datasets (for example, hand sketches or crowd sourced digital maps). While there have been many spatial calculi previously proposed (as discussed in the introduction), $L D$ is unique in allowing indeterminate directions which we believe are crucial in practice. Moreover, many previous spatial calculi have not been treated to the same theoretical analysis that we do here (i.e. the soundness, completeness and complexity results in this paper). In future work, we plan to combine the logics for qualitative distances [Du et al., 2013; Du and Alechina, 2016] and qualitative directions, and develop reasoners for checking the consistency of matching relations automatically.

We also plan to experiment with the logic on actual data in a variety of possible application scenarios. One such scenario could be in spatial data fusion. E.g. consider Figure 2; this shows detections of possible 'events' (such as a karst or an anthropomorphic structure) ahead of a Tunnel Boring Machine (TBM) from sensors mounted on the front of the TBM at different times and spatial locations as the TBM advances through the ground. The detected events will typically appear at different absolute spatial locations because as the TBM advances the sensors are better able to detect and localise features - sensors only ever give approximate locations. The challenge is to determine which events at the different time points correspond. The relative positions/directions of the

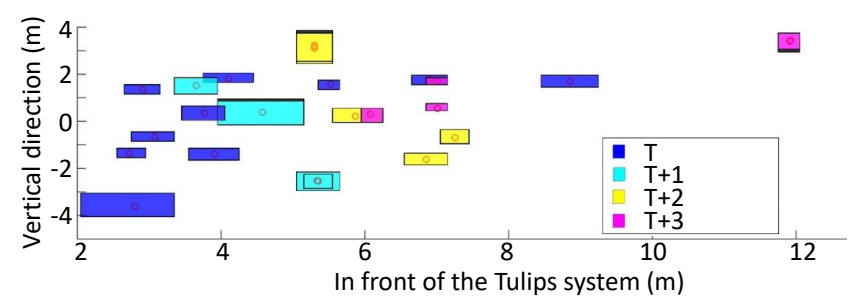

Figure 2: Detected events (rectangles) and their centroids (circles within the rectangles) at different times ahead of a TBM (from [Wei et al., 2019]; best viewed in colour).

events can be represented using $L D$. (Of course $L D$ is a logic of points, not regions, but for the purposes of this example we can use the centroid or, probably better, the end points, or just the nearest endpoint since that will have best signal.) In [Wei et al., 2019] simple overlap is used to decide whether two events are the same or not. We hypothesize that it is possible to build a more nuanced system using $L D$. Events which are $d E$ or $d W$ of each other, may be regarded as discrete events; but if they are $n E W$ then they are candidates to be the same event. By varying $\sigma$ and $\tau$ different levels of tolerance and indeterminacy could be considered and presented to the TBM experts for further analysis and verification.

\section{Acknowledgements}

This work is supported by the Young Scientist programme of the National Natural Science Foundation of China (NSFC) with a project code 61703218 . Anthony Cohn is partially supported by a Fellowship from the Alan Turing Institute, and by the EU Horizon 2020 under grant agreement 825619.

\section{References}

[Aiello et al., 2007] Marco Aiello, Ian Pratt-Hartmann, and Johan van Benthem, editors. Handbook of Spatial Logics. Springer, 2007.

[Allen, 1983] James F. Allen. Maintaining Knowledge about Temporal Intervals. Communications of the ACM, 26(11):832-843, 1983.

[Baader and Peñaloza, 2010] Franz Baader and Rafael Peñaloza. Axiom Pinpointing in General Tableaux. Journal of Logic and Computation, 20(1):5-34, 2010.

[Balbiani et al., 1998] Philippe Balbiani, Jean-François Condotta, and Luis Fariñas del Cerro. A Model for Reasoning about Bidimensional Temporal Relations. In Proc. 6th Int. Conf. on Principles of Knowledge Representation and Reasoning (KR'98), pages 124-130, 1998.

[Balbiani et al., 2007] Philippe Balbiani, Valentin Goranko, Ruaan Kellerman, and Dimiter Vakarelov. Logical Theories for Fragments of Elementary Geometry. In Marco Aiello, Ian Pratt-Hartmann, and Johan van Benthem, editors, Handbook of Spatial Logics, pages 343-428. Springer, 2007.

[Billen and Clementini, 2004] Roland Billen and Eliseo Clementini. A Model for Ternary Projective Relations 
between Regions. In Proc. 9th Int. Conf. on Extending Database Technology (EDBT), pages 310-328, 2004.

[Cohen-Solal et al., 2015] Quentin Cohen-Solal, Maroua Bouzid, and Alexandre Niveau. An Algebra of Granular Temporal Relations for Qualitative Reasoning. In Proc. 24th Int. J. Conf on AI (IJCAI), pages 2869-2875, 2015.

[Du and Alechina, 2016] Heshan Du and Natasha Alechina. Qualitative Spatial Logics for Buffered Geometries. Journal of Artificial Intelligence Research, 56:693-745, 2016.

[Du et al., 2013] Heshan Du, Natasha Alechina, Kristin Stock, and Michael Jackson. The Logic of NEAR and FAR. In Proceedings of the 11th International Conference on Spatial Information Theory, volume 8116 of LNCS, pages 475-494. Springer, 2013.

[Du et al., 2015] Heshan Du, Hai Nguyen, Natasha Alechina, Brian Logan, Michael Jackson, and John Goodwin. Using Qualitative Spatial Logic for Validating Crowd-Sourced Geospatial Data. In Proceedings of the 27th Conference on IAAI, pages 3948-3953, 2015.

[Freksa, 1992] Christian Freksa. Using orientation information for qualitative spatial reasoning. In Proc. Theories and Methods of Spatio-Temporal Reasoning in Geographic Space, Int. Conf. GIS, pages 162-178, 1992.

[Goyal and Egenhofer, 1997] Roop K. Goyal and Max J. Egenhofer. The Direction-Relation Matrix: A Representation for Direction Relations between Extended Spatial Objects. In The Annual Assembly and the Summer Retreat of Univ. Consortium for Geog. INf. Systems Science, 1997.

[Jonsson and Bäckström, 1998] Peter Jonsson and Christer Bäckström. A Unifying Approach to Temporal Constraint Reasoning. Artificial Intelligence, 102(1):143-155, 1998.

[Kreutzmann and Wolter, 2014] Arne Kreutzmann and Diedrich Wolter. Qualitative Spatial and Temporal Reasoning with AND/OR Linear Programming. In Proceedings of the 21st European Conference on Artificial Intelligence (ECAI), volume 263, pages 495-500, 2014.

[Ligozat, 1993] Gérard Ligozat. Qualitative Triangulation for Spatial Reasoning. In Proceedings of the 1st International Conference on Spatial Information Theory (COSIT), pages 54-68, 1993.

[Ligozat, 1998] Gérard Ligozat. Reasoning about Cardinal Directions. Journal of Visual Languages \& Computing, 9(1):23-44, 1998.

[Ligozat, 2012] Gérard Ligozat. Qualitative Spatial and Temporal Reasoning. ISTE Ltd and J. Wiley \& Sons, 2012.

[Litvintchouk and Pratt, 1977] Steven D. Litvintchouk and Vaughan R. Pratt. A Proof-Checker for Dynamic Logic. In Proc. 5th Int. J. Conf. on AI (IJCAI), pages 552-558, 1977.

[Marx and Reynolds, 1999] Maarten Marx and Mark Reynolds. Undecidability of Compass Logic. Journal of Logic and Computation, 9(6):897-914, 1999.

[Montanari et al., 2009] Angelo Montanari, Gabriele Puppis, and Pietro Sala. A Decidable Spatial Logic with ConeShaped Cardinal Directions. In Proc.23rd Int. Workshop of Computer Science Logic, volume 5771 of LNCS, pages 394-408, 2009.

[Morales et al., 2007] Antonio Morales, Isabel Navarrete, and Guido Sciavicco. A new modal logic for reasoning about space: spatial propositional neighborhood logic. Ann. Math. Artif. Intell., 51(1):1-25, 2007.

[Pratt, 1977] Vaughan R. Pratt. Two easy theories whose combination is hard. Technical report, Massachusetts Institute of Technology, 1977.

[Renz and Mitra, 2004] Jochen Renz and Debasis Mitra. Qualitative Direction Calculi with Arbitrary Granularity. In Proceedings of the 8th Pacific Rim International Conference on Artificial Intelligence, pages 65-74, 2004.

[Scivos and Nebel, 2004] Alexander Scivos and Bernhard Nebel. The Finest of its Class: The Natural Point-Based Ternary Calculus LR for Qualitative Spatial Reasoning. In Proc. Int. Conf Spatial Cognition, pages 283-303, 2004.

[Shostak, 1981] Robert E. Shostak. Deciding Linear Inequalities by Computing Loop Residues. Journal of the ACM, 28(4):769-779, 1981.

[Skiadopoulos and Koubarakis, 2004] Spiros Skiadopoulos and Manolis Koubarakis. Composing cardinal direction relations. Artificial Intelligence, 152(2):143-171, 2004.

[Skiadopoulos and Koubarakis, 2005] Spiros Skiadopoulos and Manolis Koubarakis. On the consistency of cardinal direction constraints. Artif. Intell., 163(1):91-135, 2005.

[Szczerba and Tarski, 1979] Lesław W. Szczerba and Alfred Tarski. Metamathematical Discussion of Some Affine Geometries. Fundam. Mathematicae, 104:155-192, 1979.

[Tarski and Givant, 1999] Alfred Tarski and Steven Givant. Tarski's system of geometry. Bulletin of Symbolic Logic, 5(2):175-214, 1999.

[Tarski, 1959] Alfred Tarski. What is Elementary Geometry? In Leon Henkin, Patrick Suppes, and Alfred Tarski, editors, The Axiomatic Method, volume 27 of Studies in Logic and the Foundations of Mathematics, pages 16-29. Elsevier, 1959.

[Trybus, 2010] Adam Trybus. An Axiom System for a Spatial Logic with Convexity. In Proc. 19th Europ. Conf. on AI (ECAI), pages 701-706, 2010.

[Vilain and Kautz, 1986] Marc B. Vilain and Henry A. Kautz. Constraint Propagation Algorithms for Temporal Reasoning. In Proceedings of the 5th National Conference on Artificial Intelligence (AAAI-86), pages 377-382, 1986.

[Walega and Zawidzki, 2019] Przemyslaw Andrzej Walega and Michal Zawidzki. A Modal Logic for SubjectOriented Spatial Reasoning. In Proc. 26th Int. Symp. on Temporal Representation and Reasoning, volume 147 of LIPICs, pages 4:1-4:22, 2019.

[Wei et al., 2019] Lijun Wei, Muhammad Khan, Owais Mehmood, Qingxu Dou, Carl Bateman, Derek R. Magee, and Anthony G. Cohn. Web-based visualisation for lookahead ground imaging in tunnel boring machines. Automation in Construction, 105:102830, 2019. 